\title{
Zentral- und Landesbibliothek Berlin (ZLB) erhält nationale Auszeichnung „Bibliothek des Jahres“ 2019
}

http://doi.org/10.1515/bd-2020-0006

Am bundesweiten „Tag der Bibliotheken“ hat die Zentral- und Landesbibliothek Berlin (ZLB) am 24.10.2019 im Festsaal des Berliner Rathauses die nationale Ehrung „Bibliothek des Jahres“ erhalten. Ausgezeichnet wurde die ZLB für ihre strategisch ausgerichteten, partizipativen Angebote und digitalen Services, mit denen sie stetig auf die gesellschaftlichen und medialen Veränderungen reagiert und sich so als öffentliches Forum in der Stadtgesellschaft etabliert hat. Der Preis, der 2019 zum 20. Mal vergeben wurde, ist mit 20.000 Euro dotiert und wird vom Deutschen Bibliotheksverband e.V. (dbv) und der Deutsche Telekom Stiftung vergeben.

Überreicht wurde die Auszeichnung von Dr. Thomas de Maizière, MdB und Vorsitzender der Deutsche Telekom Stiftung, sowie von Dr. Frank Mentrup, Präsident des Deutschen Bibliotheksverbandes und Oberbürgermeister der Stadt Karlsruhe.

Im Rahmen der Verleihung sagte Dr. Thomas de Maizière, MdB: „Videos streamen, digitale Lese- und Hörmedien sowie E-Learning - mit ihrem Projekt, Digitale Welten' hat die Zentral- und Landesbibliothek Berlin ein attraktives, zukunftsgerichtetes Angebot geschaffen, insbesondere für Nutzer, die sonst nicht viele Bücher lesen. Das alles hat Vorbildcharakter.“

Dr. Frank Mentrup hob die ZLB als sozialen Ort hervor: „Die Zentral- und Landesbibliothek Berlin ist nicht nur ein Wissensspeicher und Informationsvermittler, sondern auch ein offener und für die gesamte Bevölkerung frei zugänglicher Ort der Bildung und Kultur. Insbesondere mit ihrem Arbeitsbereich ,CommunityProjekte' schafft sie es, gemeinsam mit Partnerinnen und Partnern sowie Bürgerinnen und Bürgern attraktive Angebote für Meinungsbildung und Meinungstausch und damit einen Ort für das demokratische Gemeinwohl zu schaffen.“

Der Vorstand der Stiftung Zentral- und Landesbibliothek Berlin, Volker Heller, dankte den Mitarbeiterinnen und Mitarbeitern der ZLB: „Die gesamte ZLB freut sich sehr über diese Ehrung. Sie zeigt, dass wir mit unserer Entwicklung der letzten Jahre auf dem richtigen Weg sind und den Berlinerinnen und Berlinern eine ganz besondere Bibliothek auf hohem Niveau bieten. Das freut mich auch ganz persönlich und ist zugleich Ansporn für unsere weitere Arbeit. Aber heute feiern wir erstmal.“ 
Im Rahmen der Verleihung ging es darüber hinaus auch um kulturpolitische Themen. So hob die Vorsitzende des Ausschusses für Kultur und Medien im Deutschen Bundestag, Katrin Budde, MdB, im Gespräch mit dem Bundesvorsitzenden des dbv, Prof. Dr. Andreas Degkwitz, die Bedeutung von Bibliotheken für digitale Teilhabe und einen demokratischen Dialog hervor. Sie sagte: „Die Zentral- und Landesbibliothek Berlin bietet nicht nur allen Bürgerinnen und Bürgern die Möglichkeit, sich analog und digital zu informieren und weiterzubilden. Die Bibliothek ist auch ein wunderbarer Ort der Begegnung und des Austausches über Kunst und Kultur“.

Im Gespräch mit den Mitarbeiterinnen und Mitarbeitern der Zentral- und Landesbibliothek Berlin sprach sich Dr. Klaus Lederer, Senator für Kultur und Europa, für den geplanten Neubau der ZLB am Blücherplatz aus: „Das Land Berlin hat den Weg frei gemacht für einen Bibliotheksstandort, der allen Ansprüchen an eine moderne, zukunftsoffene und sehr lebendige Bibliothek des 21. Jahrhunderts gerecht wird. Die Zusammenführung der ZLB am Standort Blücherplatz setzt ein deutliches Zeichen für die kulturelle Bildung und lebenslanges Lernen in Berlin. Nichts ist heute so wichtig wie Wissen und dessen Anwendung in Vernetzung, Austausch und Begegnung. Die Zeit von ,Buchausleihstationen' ist vorbei - die neue ZLB wird das Wohnzimmer der Stadtgesellschaft.“

Weitere Informationen zur „Bibliothek des Jahres“ 2019 finden Sie hier.

\section{Der Deutsche Bibliotheksverband e.V. (dbv)}

Der Deutsche Bibliotheksverband e.V. (dbv) vertritt mit seinen mehr als 2.100 Mitgliedern bundesweit rund 10.000 Bibliotheken mit 25.000 Beschäftigten und 11 Mio. Nutzer^innen. Sein zentrales Anliegen ist es, Bibliotheken zu stärken, damit sie allen Bürger`innen freien Zugang zu Informationen ermöglichen. Der Verband setzt sich ein für die Entwicklung innovativer Bibliotheksleistungen für Wissenschaft und Gesellschaft. Als politische Interessensvertretung unterstützt der dbv die Bibliotheken, insbesondere auf den Feldern Informationskompetenz und Medienbildung, Leseförderung und bei der Ermöglichung kultureller und gesellschaftlicher Teilhabe für alle Bürger`innen.

1 https://www.bibliotheksverband.de/dbv/presse/presse-details/archive/2019/may/article/ forum-fuer-die-stadtgesellschaft-die-zentral-und-landesbibliothek-berlin-ist-bibliothekdes-j.html?tx_ttnews\%5Bday\%5D=22\&cHash=694c64c80014edf7bbb96458fd26cd05 [Zugriff: 24.10.2019]. 


\section{Die Deutsche Telekom Stiftung}

Die Deutsche Telekom Stiftung wurde 2003 gegründet, um den Bildungs-, Forschungs- und Technologiestandort Deutschland zu stärken. Mit einem Kapital von 150 Millionen Euro gehört sie zu den großen Unternehmensstiftungen in Deutschland. Die Stiftung unterstützt gezielt Projekte, die sich an Kinder und Jugendliche im Alter von 10 bis 16 Jahren richten und sich mit Themen aus dem mathematisch-naturwissenschaftlich-technischen Umfeld beschäftigen.

\section{Pressekontakte:}

Zentral- und Landesbibliothek Berlin (ZLB)

Anna Jacobi, Pressesprecherin

Tel.: 030 / 90226-598, E-Mail: anna.jacobi@zlb.de

URL: https://www.zlb.de

Deutscher Bibliotheksverband e.V. (dbv)

Kristin Bäßler, Leitung Kommunikation / Pressesprecherin

Tel.: 030 / 6449899 25, E-Mail: presse@bibliotheksverband.de

URL: www.bibliotheksverband.de

\section{Deutsche Telekom Stiftung}

Matthias Adam, Referent Kommunikation

Tel.: 0228 / 181-92015, E-Mail: matthias.adam@telekom-stiftung.de

URL: https://www.telekom-stiftung.de 